\title{
EUROPEAN SOCIETY FOR PAEDIATRIC INFECTIOUS DISEASES
}

\author{
Selected Abstracts from the 6th Annual Meeting \\ April 27-29, 1988-Padua, Italy
}

\section{Officers}

\section{CHAIRMAN}

Ruggiero D'Elia, Italy

TREASURER

Volker von Loewenich, West Germany

SECRETARY

John de Louvois, England

VICE-CHAIRMAN

Jacomina Hoogkamp-Korstanje, Holland

\section{Board Members}

Ron Dagan, Israel

Immaculada Puig de la Capilla, Spain

CHAIRMAN ELECT

Jacomina Hoogkamp-Korstanje, Holland

VICE-CHAIRMAN ELECT

Berge Trollfors, Sweden

PRESENT SECRETARY

Helen Holzel

Dept of Microbiology

Hospital for Sick Children

Great Ormond Street, London WC1

THE SURVEILLANCE OF RARE PAEDIATRIC INFECTIONS AND INFECTION-RELATED DISORDERS Susan Hall, Public 1 Health Laboratory Service, Communicable Disease Surveillance Centre (CDSC) London.

Epidemiological surveillance of rare disorders eg. Kawasaki Disease (KD), paediatric AIDS, differs from that of common infections such as measles; very large base popul-ations and maximal sensitivity and specificity of case ascertainment are required. These depend on: disease severity (patient presents for medical care); diagnostic awareness; sensitivity and specificity of the case definition; availability of and expertise in sophisticated laboratory investigations: knowledge of and willingness to report to, the surveillance scheme; careful follow-up to ensure satisfaction of case criteria and to exclude duplicates and other errors. The of case criteriatric surveillance Unit (BPSU) De British Paediatric Association, CDSC London, Institute of (hild Health), a new active case ascertainment scheme to facilitate the study of rare paediatric disorders, began operation in July 1986 . A report card is sent monthly to the 800 senior paediatricians throughout the British Isles. Respondents indicate, whether in the previous calendar month, they have seen a patient with one of the reportable conditions listed on the card; nil returns are requested. 'Positive' replies are forwarded to the research workers concerned and currently the scheme is being used to survey 11 rare disorders including AIDS, KD, Reyeing used to surva neonatal herpes, SSPE \& haemolytic uraemic syndrome. The BPSU promotes the ideals of rare disease surveillance with its large base population, high response rate (88-90\%) and an active case gathering method, which maximises sensitivity.

HIV IN PAEDIATRICS-THE EUROPEAN COLLABORATIVE STUDY

2 Dept of Paediatric Epidemiology, London WC1N 1EH,

England

The objectives of the ECS are to determine:-

1. the prevalance of HIV infection in infanats born to positive mothers; 2 . the risk factors influencing transmission to infants; 3 . the natural history of HIV infection in infants; 4. precursors of AIDS/ARC onset in infected infants. Seven centres are participating; Padua, Edinburgh, Berlin, Madrid, Valencia and Amsterdam. Seropositive women are identified in the antenatal period and the paediatrician is notified. intervals. By $31 / 3 / 88204$ children, mean birth wt. $2800 \mathrm{~g}$ mean gest. age 38 wks, had been enrolled into the study. $85 \%$ of mothers were IV drug abusers; $25 \%$ of babies had drug withdrwal symptoms. 122 children have been followed for at least 6 months and 89 for over 1 year. The median age at last visit was 12 months 10 children 5 had died of opportunistic infections; 3 premature infants died in the neonatal period. 18 infants had nonspecific signs or symptoms. The majority $173 / 204$ were clinically normal when symptoms. The majority $173 / 204$ were clinically normal when last seen. No cases of AIDS dysmorphic syndrome were described.
$9 / 65(14 \%)$ children over 15 months remain antibody positive. 4 other children died of AIDS/ARC who would have been over 15 months had they survived. Several children have lost antibody despite being virus and/or antigen positive. At present the numbers are too small for meaningful estimates of risk factors in relation to infection to be calculated. There have been no side effects with any immunisations.
EPIOEMIOLOGY OF PEDIATRIC HIV INFECTION IN ITALY 3 MDe Martino ${ }^{* \star \star}$ Dept of Pediatrics, University of Padova; ${ }^{\star x}$ Dept of Pediatrics, University Turin; ***0spedale Meyer, Firenze, I taly.

By April 1988, $955(1.5 \%)$ children with AIDS had been reported to the U.S. Centers for Disease Control (CDC). Some had received blood transfusions, but $77 \%$ were born to mothers at risk of AIDS. In Italy the prevalence of HIV infection among intravenous drug users ranges from $40-80 \%$ in different urban areas, and is higher than in most European countries. Although the definition of pediatric AIDS is limited and does not cover important aspects of symptomatic HIV infection, in Italy children with full-blown AIDS represent $3 \%$ of all reporter cases. In 1985 an Italian Register for pediatric HIV infection was activatd by the Italian Pediatric Society. By March 1988, 544 children "at risk" were reported, $485(90 \%)$ were born to seropositive mothers and the remaining children acquired born to seropositive mothers and the remaining children acquired
the infection through transfusions. The distribution of children who acquired the infection from mother follows that of drug addiction in the country; most of the children who acquired the infection through blood transfusion (often b-Thalassemic) were observed in Sardinia According to the CDC classification 165 children born to HIV positive mothers are "infected" 92 "not infected" and 228 "indeterminate". Over $80 \%$ of infected 92 "not (nfected patients were symptomatic symptoms, have some immunological modifications. Symptoms usually appear in the first year of life. In USA $60 \%$ of children born to HIV positive mothers are in foster care or adopted, in Italy more than $80 \%$ live with their natural families.

\section{PAEDIATRIC HIV-STUDY ZURICH: FIRST RESULTS \\ 4 David Nadal, U Hunziker, Z Tomasik, J-C Wet

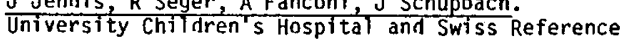 \\ Center for Retrovirus, Zurich, Switzerland} Since June 1986 thirty-two children born to HIV-
positive mothers have been followed at the AIDS-outpatient positive mothers have been followed at the AIDS-outpatient
clinic of the Children's Hospital Zurich. They were given 3 month check-ups which included neurodevelopmental, immunological and virological evaluation. The main purpose of our longitudinal study is the assessment of immunological and microbiological trigger factors of an HIV-encephalopathy. Six of the 32 children are definitely infected with HIV as demonstrated by culture, 5 of them have developed an immunodeficiency and 2 are symptomatic (encephalopathy). Four of 32 are possibly infected with HIV (positive specific IgM and/or IgA), one of them has an immunodeficiency and a second and/or IgA), one of them has an immunodeficiency and a second shows psychomotor retardation. In 22 children, infection with
HIV can neither be ascertained nor disproven. One of them is immunodeficient, another presents a spastic hemiparesis. Up to now, our data reveal a close correlation between positive HIV culture and immunodeficiency on one hand, and HIV-associated symptoms including encephalopathy on the other. However, a negative HIV-culture together with immunocompetence are not associated with clinical symptoms. The risk for developing AIDS remains uncertain in those children in whom only HIV-anatibodies or an immunodeficiency have been revealed. 